\title{
Comparison of PapilloCheck and Linear Array to Detect and Differentiate Human Papillomaviruses in Cervical and Tonsillar Smears from Females with Cervical Intraepithelial Lesions
}

\author{
Donata Grimm $^{1 *}$, Linn Woelber ${ }^{1}$, Katharina Prieske ${ }^{1}$, Barbara Schmalfeldt ${ }^{1}$, Sascha Kürti ${ }^{1}$, \\ Chia-Jung Busch $^{3}$, Ingo Teudt ${ }^{4,5}$, Oliver Brummer ${ }^{6}$, Volkmar Mueller ${ }^{1}$ and Thomas Meyer ${ }^{2}$ \\ 'Departmentof Gynaecology and Gynaecologic Oncology, University Medical Center Hamburg-Eppendorf, Germany \\ 2 Departmentof Medical Microbiology, Virology and Hygiene, University Medical Center Hamburg-Eppendorf, Germany \\ ${ }^{3}$ Departmentof Otorhinolaryngology, Head and Neck Surgery and Oncology, University Medical Centre Hamburg-Eppendorf, Germany \\ ${ }^{4}$ Departmentof Otolaryngology, Head and Neck Surgery, Asklepios Klinik Hamburg Altona, Germany \\ HNO-in-Altona,Private Praxis of Otorhinolaryngology, Hamburg, Germany \\ ${ }^{6}$ TagesklinikAltonaer Strasse, Private Praxis of Gynaecology, Hamburg, Germany
}

Received: 10 July 2018; accepted: 18 July 2018

\begin{abstract}
A subgroup of oropharyngeal squamous cell carcinomas (OSCCs) are causally linked to infection with high-risk human papillomaviruses (HR-HPVs). To evaluate the prevalence of simultaneous oral HPV infection in females with cervical high-grade squamous intraepithelial lesions (HSIL), tonsillar- and cervical smears were collected simultaneously from 73 patients and analyzed for HPV using two commercial assays, PapilloCheck (Greiner-BioOne) and Linear Array (Roche). Only 3/73 (4.1\%) tonsillar smears were HPV positive (HPV+), with HPV types 16,35 , and 45 , respectively, detected by both assays ( $100 \%$ agreement). Concordant results were also found in $60 / 66(91 \%)$ evaluable cervical smears. Of specimens, positive by both assays, typing results completely coincide in $71 \%$ (all types are identical) and partially coincide in $27 \%$ (at least one type is identical). Taken together, results of HPV detection and typing by PapilloCheck and Linear Array are highly congruent and confirm the low prevalence of HR-HPV in tonsillar smears of patients with HSIL of the uterine cervix. Our data indicate low prevalence of oropharyngeal HPV infection in patients with high-grade cervical dysplasia. The low detection rate was confirmed by using two different commercial assays with largely consistent results of HPV detection and typing, but with some variation for particular HPV types. Comparative testing of larger numbers is required to identify the HPV types prone to escape detection with particular assays.
\end{abstract}

Keywords: human papillomavirus (HPV), oropharyngeal squamous cell carcinoma (OSCC), sexual transmitted disease, CIN, HSIL, cervical cancer (CC)

\section{Introduction}

Oncogenic human papillomaviruses (HPVs) are strongly associated with dysplastic and neoplastic epithelial lesions and are considered a necessary prerequisite for the development of cervical cancer (CC) and its precursor lesions [1]. Accordingly, 12 so-called high-risk (HR) HPV types (16, 18, 31, 33, $35,39,45,51,52,56,58$, and 59) are classified as group 1 agents, carcinogenic to humans according to the International Agency for Research on Cancer (IARC). Increased oncogenic potential is also ascribed to HPV 68 and 73 [2].

Among these HR-HPV types, the highest risk of malignant transformation is described for persistent infection with HPV 16 and HPV 18 [3, 4]. In addition to CC, HR-HPV were also detected in $40-60 \%$ of vulvar cancers, $70 \%$ of vaginal cancers, $50 \%$ of penile cancers, $80-90 \%$ of anal cancers, and in about one third of oropharyngeal cancers, in particular affecting the tonsils and the bottom of the tongue [2].

Approximately $80 \%$ of the human population is expected to acquire mucosal HPV infection once in a lifetime. The highest rate of infection is seen in young adults aged 20-30 years.

\footnotetext{
*Author for correspondence: Department of Gynaecology and Gynaecologic Oncology, University Medical Center Hamburg-Eppendorf, Martinistraße 52, 20246 Hamburg, Germany; Tel: +49 407410 52510; Fax: +49407410 40070; E-mail: d.grimm@uke.de.
}

More than $90 \%$ of immunocompetent persons will eliminate the virus within 24 months [5]. Thus, in most cases, mucosal HPV infection does not persist for long times and does not induce (pre)malignant epithelial lesions. On the other hand, patients with high-grade cervical intraepithelial neoplasia (CIN) may be considered unable to control or eliminate cervical HRHPV infection. Indeed, these patients show insufficient cellular immune reactions to HR-HPV oncogenes, in contrast to patients that spontaneously resolve HR-HPV infection [5]. Moreover, failure to control cervical HR-HPV infection may also apply for other mucosal tissues. Depending on sexual behavior, females with CIN may therefore be at higher risk of having oral HPV infection.

According to a systematic review combining the results of 18 studies about oral HPV infection, a similar prevalence of $4.6 \%$ and $4.4 \%$ was calculated for healthy males and females, but the prevalence data varied significantly among studies, ranging from $0.6 \%$ to $20.7 \%$ [6]. In a recent study on the prevalence of oral HPV infection in women screened for cervical infection, no correlation of cervical and oral HPV infection was observed [7]. Again, similar low rates of $5.7 \%$ and $5.1 \%$ for oral HPV infection were found in females with and without cervical HPV infection, respectively. However, no information about the persistence of HPV infection was given, and cervical dysplasia that may indicate persistent

This is an open-access article distributed under the terms of the Creative Commons Attribution-NonCommercial 4.0 International License (https://creativecommons.org/licenses/by-nc/4.0/), which permits unrestricted use, distribution, and reproduction in any medium for non-commercial purposes, provided the original author and source are credited, a link to the CC License is provided, and changes - if any - are indicated. 
HPV infection was reported in less than $20 \%$ of the females included.

We recently performed a study to analyze oral HPV infection in patients with histologically confirmed high-grade CIN (high-grade squamous intraepithelial lesions [HSIL]), which usually results from persistent HR-HPV infection, and also detected HPV infrequently (4.4\%) in oropharyngeal specimens [8]. In order to assess the comparability of test results of different assays and to confirm the low prevalence in oral specimens, cervical and oral specimens from a subset of patients were analyzed with two different commercial assays for HPV detection and typing (PapilloCheck, GBO, based on hybridization using DNA chip technology and HPV Linear Array, Roche, based on reverse line blot hybridization).

\section{Material and Methods}

Patient Cohort. In 2014, we conducted a prospective bicentric cohort study of the University Medical Center Hamburg-Eppendorf in collaboration with the Medical Center Asklepios Altona to determine the prevalence of oropharyngeal HPV infection in women with cervical HSIL. Women with abnormal Pap smear were admitted to the colposcopy units of both clinics, and swabs were taken from their uterine cervix and tonsils to test for cervical and oral HPV infection. Inclusion criteria were high-grade CIN lesions (confirmed by histology) and a minimum age of 18 years. Exclusion criteria were other not-HPV-related diseases of the vulva, vagina, and cervix, malignant diseases of the cervix, previous tonsillectomy, or pregnancy.

A standardized questionnaire was used to record data collected in an interview focusing on demographic characteristics, tobacco and alcohol use, clinical history of sexually transmitted diseases, sexual activity, sexual behaviors, and contraception methods.

Clinical Samples. Cervical smears containing superficial cells were taken from the ecto- and the endocervix by using a cytobrush (Gynobrush $^{\circledR}$, Heinz Herenz Hamburg, Germany). For tonsillar sampling, the PapCone ${ }^{\circledR}$ device was used (Otto Bock PUR Life Science GmbH, Duderstadt, Germany) to collect superficial scrapes of the mucosa of the tonsils, by performing 5-10 complete backward and forward brushes at both the right and left tonsil. Cell material from the brushes was transferred to vials with viral transport medium.

HPV Detection and Typing. From 73 women, both cervical and tonsillar swabs were obtained and used for HPV testing with two commercial test kits: PapilloCheck (Greiner Bio One - GBO, Frickenhausen, Germany) and Linear Array HPV Genotyping/Detection Kit (Roche, Basel, Switzerland). The specimens were first used for HPV testing by Linear Array. Remaining DNA extracts were stored frozen at $-20{ }^{\circ} \mathrm{C}$. Due to the low detection rate of HPV in tonsillar swabs, the PapilloCheck assays were performed as a second nucleic acid amplification test (NAAT) to verify the results of the Linear Array. The two assays differ with respect to the number of HPV types detected (see Table 1). PapilloCheck and HPV Linear Array detect and differentiate 24 and 37 HPV types, respectively, with some types included exclusively in one of the tests but all HR-HPV types covered by both tests.

DNA isolation was performed using a Maxwell 16 LEV Blood DNA kit (Promega, Wisconsin, USA) on the Maxwell 16 instrument (Promega, Wisconsin, USA). From both cervical and tonsillar brushes, $200 \mu \mathrm{L}$ cell suspension was combined with $200-\mu \mathrm{L}$ lysis buffer and $20-\mu \mathrm{L}$ proteinase $\mathrm{K}$, shortly mixed by pulse-vortexing and centrifuged in a microcentrifuge to remove drops from the inside of the lid. After a 20-min incubation period at $56{ }^{\circ} \mathrm{C}$, the lysed samples were transferred into the cartridge of the DNA extraction kit for processing according to the manufacturer's protocol. The purified DNA was eluted into small sample tubes with $100-\mu l$ elution buffer.

PapilloCheck Assay. Five microliters of isolated DNA were used for amplification in a total volume of $25 \mu \mathrm{L}$ using HPV E1 primers resulting in $350 \mathrm{bp}$ PCR products, as described in the GBO instruction manual. Then, amplification products were hybridized to complementary DNA probes on the PapilloCheck DNA chip. During hybridization, the fluorescence labeling is introduced, and fluorescent signals of bound amplicons were scanned and evaluated using the CheckScanner and CheckReport Software according to the manufacturer's instructions. A fragment of human adenosine deaminase 1 (ADAT-1) gene is amplified in each sample as an internal control to monitor DNA preparation and template integrity. To control the hybridization reaction, the hybridization buffer contains a probe labeled with a different fluorophore complementary to adequate DNA probes spotted on the chip.

HPV Linear Array. PCR was performed with $50 \mu \mathrm{L}$ of the isolated DNA in a final volume of $100 \mu \mathrm{L}$ using HPV L1 primer resulting in $450 \mathrm{bp}$ PCR products according to the manufacturer's instructions [9]. After amplification, the biotinylated PCR products were denatured by addition of $100 \mu \mathrm{L} \mathrm{NaOH}$, and $75 \mu \mathrm{L}$ of the denatured amplicons were used for hybridization to identify HPV genotypes using the Linear Array protocol. To control DNA extraction and PCR efficiency, a 268-bp fragment of the human beta-globin gene was co-amplified in each sample.

Statistical Analysis. To evaluate the concordance of HPV typing by PapilloCheck and Linear Array, Cohen-Kappa values were calculated. The level of concordance was categorized as very good (kappa 0.8-1.0), good (kappa 0.6-0.8) moderate (kappa 0.4-0.6), fair (kappa 0.2-0.4), and poor (kappa <0.2) [10].

\section{Ethics}

Ethical approval was obtained from the medical association Hamburg (reference number PV438). Written informed consent was obtained from each patient prior to participation in the study.

\section{Results}

Cervical and tonsillar swabs from 73 women were tested for HPV using both PapilloCheck and HPV Linear Array.

Table 1. HPV types detected by PapilloCheck and Linear Array

\begin{tabular}{|c|c|}
\hline Test & HPV types included \\
\hline $\begin{array}{l}\text { PapilloCheck } \\
\text { (Greiner-Bio-One) }\end{array}$ & $6,11,16,18,31,33,35,39,40,42,43,44,45,51,52,53,56,58,59,66,68,70,73,82$ \\
\hline $\begin{array}{l}\text { Linear Array } \\
\text { HPV genotyping/detection kit (Roche) }\end{array}$ & $\begin{array}{c}6,11,16,18, \mathbf{2 6}, 31,33,35,39,40,42,45,51,52,53, \mathbf{5 4}, 55^{a}, 56,58,59, \mathbf{6 1}, \mathbf{6 2}, \mathbf{6 4}, 66 \\
\mathbf{6 7}, \mathbf{6 8}, \mathbf{6 9}, 70, \mathbf{7 1}, \mathbf{7 2}, \mathbf{7 3}, \mathbf{8 1}, 82, \mathbf{8 3}, \mathbf{8 4}, \mathbf{8 9} \mathbf{9}^{b}, \text { IS } 39\end{array}$ \\
\hline
\end{tabular}


Table 2. Concordance of HPV detection with PapilloCheck and Linear Array in cervical smears

\begin{tabular}{lcc}
\hline Cervical smears $(n=66)$ & PapilloCheck positive & PapilloCheck negative \\
\hline Linear Array positive & 59 & 3 \\
Linear Array negative & 3 & 1 \\
\hline
\end{tabular}

Patients were 22-66 years old (average 32.6 years), and all had histologically confirmed HSIL. The HPV results of all specimens are summarized in the supplementary data (Table S1). Using the HPV Linear Array, HPV was detected in 69/73 cervical swabs. Among the 4 specimens in which no HPV was detected, 3 were positive by PapilloCheck (identified HPV types: 16, 31, and 33, respectively).

By using PapilloCheck, HPV DNA was detected in 62/73 cervical swabs. The test was also negative in 4 specimens. In 3 of them, HPV was detected by HPV Linear Array, which identified HPV types 16, 18, and a 54/IS39 co-infection, respectively, in these cases. HPV 54 and IS39, however, are not included in the PapilloCheck assay. The results of the other 7 specimens were invalid due to the lack of amplification of the human control target, indicating insufficient cell material. Excluding invalid results of PapilloCheck, the concordance of Linear Array and PapilloCheck results was 60/66 (91\%) (Table 2). Excluding the specimen positive for HPV types 54 and IS39 that cannot be detected by PapilloCheck, the concordance increased to $60 / 65(92.3 \%)$.

HPV DNA was detected in 3/73 (4.1\%) tonsillar swabs with both PapilloCheck and HPV Linear Array. The two assays identified the same HPV types (HPV 16, 35, and 45, respectively). These HPV types were also detected in the corresponding cervical specimens. Seventy and 61 tonsillar swabs were negative by the Linear Array and PapilloCheck. In 9 tonsillar swabs, the PapilloCheck result was invalid due to the lack of amplification of the human control target, indicating insufficient cell material. Excluding these specimens, PapilloCheck and Linear Array show 100\% agreement of HPV detection in tonsillar swabs (Table 3).

In summary, detection of HPV by PapilloCheck and Linear Array coincides in 124 of 130 tonsillar or cervical smears (concordance 95.4\%, kappa 0.70). Within the data about sexual behavior recorded during the interview included in the study, frequent or occasional oral sex was stated by all three patients with HPV positive tonsillar smears, as well as by 63 / $70(90 \%)$ patients with HPV negative tonsillar smears. In addition, the 3 patients with oral HPV infection were smokers ( $>5$ cigarettes per day), whereas $50 \%$ of the patients with HPV negative tonsillar smears were non-smokers.

Further, we compared the identified HPV types in specimens positive by both assays. In all 35 specimens with one HPV type (3 tonsillar swabs and 32 cervical swabs), the PapilloCheck and Linear Array results were identical (100\% agreement). In the other 27 specimens (all cervical swabs), more than one HPV type was identified with at least one assay. When excluding HPV types not included in the panels of PapilloCheck or Linear Array, the same HPV types were identified in 9 of them, and at least one HPV type was identical in 17 of them. In only one specimen, the HPV types identified were totally different.

Table 3. Concordance of HPV detection with PapilloCheck and Linear Array in tonsillar smears

\begin{tabular}{lcc}
\hline $\begin{array}{l}\text { Tonsillar smears } \\
(n=64)\end{array}$ & $\begin{array}{c}\text { PapilloCheck } \\
\text { positive }\end{array}$ & $\begin{array}{c}\text { PapilloCheck } \\
\text { negative }\end{array}$ \\
\hline Linear Array positive & 3 & 0 \\
Linear Array negative & 0 & 61 \\
\hline
\end{tabular}

Table 4. Agreement of HR-HPV typing by PapilloCheck and Linear Array

\begin{tabular}{lcccc}
\hline $\begin{array}{l}\text { HPV } \\
\text { type }\end{array}$ & $\begin{array}{c}\text { Detected by } \\
\text { PapilloCheck }\end{array}$ & $\begin{array}{c}\text { Detected by } \\
\text { Linear Array }\end{array}$ & $\begin{array}{c}\text { Detected by } \\
\text { PapilloCheck or } \\
\text { Linear Array }\end{array}$ & $\begin{array}{c}\text { Kappa } \\
\text { value }\end{array}$ \\
\hline 16 & 31 & 31 & 32 & 0.96 \\
18 & 3 & 5 & 6 & 0.47 \\
31 & 11 & 9 & 12 & 0.78 \\
33 & 3 & 2 & 3 & nd $^{a}$ \\
35 & 2 & 2 & 2 & $\mathrm{nd}$ \\
39 & 3 & 3 & 3 & $\mathrm{nd}$ \\
45 & 3 & 4 & 4 & 0.85 \\
51 & 5 & 4 & 5 & 0.88 \\
52 & 5 & 2 & 5 & 0.56 \\
56 & 5 & 3 & 5 & 0.75 \\
58 & 5 & 5 & 1 & nd \\
59 & 1 & 1 & 2 & nd \\
68 & 2 & 1 & & \\
\multicolumn{2}{l}{${ }^{a}$ nd: not determined. } & & &
\end{tabular}

nd: not determined.

Taken together, typing results of PapilloCheck and Linear Array were fully consistent in 44/62 (71\%) and partially consistent (at least one type identical) in 17/62 (27\%). The agreement of Linear Array and PapilloCheck test results for particular HPV types was further assessed by calculating Cohens Kappa values for individual HR-HPV types (Table 4). Considering HPV types detected by any test in at least 4 specimens, a high level of concordance was found for HPV types $16,45,51$, and 58 , as indicated by kappa $>0.8$. Concordance was good for HPV types 31 and 56 with kappa of $0.61-0.8$, but only moderate for HPV 18 and 52 (kappa 0.4-0.6).

\section{Discussion}

In this study on female patients with high-grade cervical lesions, the rate of HR-HPV in tonsillar swabs was only $4.1 \%$, indicating a low prevalence of oropharyngeal HPV infection in these patients. The low frequency of HPV detection was confirmed by using two commercial assays for HPV detection and typing (PapilloCheck and Linear Array) that have been shown to be suitable for HPV diagnosis in global proficiency studies [11]. The results of testing tonsillar swabs obtained with the two assays showed $100 \%$ agreement, as the same 3 HR-HPV types (HPV 16, 35 and 45, respectively) were identified in the same three patients. These HPV types were also detected in the corresponding cervical smears, indicating that these patients may generally fail to immunologically eliminate the virus [12]. All three participants stated occasional oral sex and smoking ( $>5$ cigarettes per day) in the study questionnaire. On the other hand, oral sex was also reported by $90 \%$ of 70 patients with HPV negative tonsillar smears, indicating potential exposure to HPV, but $50 \%$ of them were non-smokers. Possible explanations for the lack of HPV detection include a lower susceptibility of oropharyngeal epithelia compared to the cervix uteri or other/additional mechanisms of HPV clearance that might be affected by smoking [13].

A potential weakness of the study relates to the oropharyngeal specimen type. Although tonsillar swabs are considered suitable for detection of oropharyngeal HPV, infection at other sites of the oral cavity (as for instance, the bottom of the tongue) may be missed. In our study we obtained additional oral lavage from another subset of 42 patients. These specimens were not available for the comparison of Linear Array and PapilloCheck, but tested by PapilloCheck only. HPV was detected in 2/42 tonsillar smears and 2/42 lavage specimens (each $4.8 \%$ ) that came from 3 different patients (from one patient both smear and lavage was positive). Thus, even when 
the results from lavage and tonsillar smears were combined, the HPV detection rate remained low (3/42 patients; $7.1 \%)$.

The reliability of HPV results in tonsillar swabs was further corroborated by the high sensitivity of both tests to detect HPV in cervical lesions and by the good agreement of typing results obtained with the two assays. The detection rate of 93.9\% (62/66) by PapilloCheck and 94.9\% (69/73) by Linear Array is in line with previously reported results. In the evaluation study, the PapilloCheck HPV genotyping test was compared with PGMY09/11 PCR line blot assay (the premarket version of the Linear Array), SPF10 PCR line probe array, and Hybrid Capture 2 (HC2). HPV DNA was detected in $98.4 \%$ of 63 cervical smears from patients with HSIL by PapilloCheck, SPF10 array, and HC2 and in $93.7 \%$ by the Linear Array prototype test [14]. A similar sensitivity of PapilloCheck and Linear Array for HSIL (96\% and 97\%, respectively) was described by Halfon et al. 2010 [15]. In a larger study comparing PapilloCheck with $\mathrm{HC} 2$ for detection of HSIL in 672 retrospectively selected samples, $89 \%$ were positive by PapilloCheck compared to $93.5 \%$ by HC2 [16]. A recent comparison of PapilloCheck with GP5/6 Luminex Kit also revealed high sensitivity of PapilloCheck that was positive in $96.1 \%$ of 102 HSIL [17].

In our study concordant results of HPV detection were found in 60/66 cervical specimens (91\%), and in all 64 tonsillar specimens, resulting in overall concordance of 124/130 (95.4\%). Similarly, Halfon et al. reported a high level of agreement of $96 \%$ when comparing results of HPV detection with PapilloCheck and Linear Array in 239 cervical samples [15]. Differences of NAATs based HPV detection and typing assay may relate to different analytical sensitivity that varies among HPV types. Comparatively low detection rates for HPV type 35 and 56 were reported for PapilloCheck and Linear Array, respectively [14]. Also, lower sensitivity for HPV Types 31 and 52 was reported for PGMY-primer used in the Linear Array, compared to SPF10 primer [18]. In our study, specimens positive only by Linear Array were represented by HPV types 16, 18, and 54/IS39, and those positive by PapilloCheck only were represented by 16, 31, and 33. HPV 54 and strain IS39, however, are not included in the PapilloCheck panel. According to a previous study, detection rates of HPV type 16 are similar with both assays, but HPV 18 is more frequently identified with Linear Array, and HPV 31 and 33 are more frequently identified with PapilloCheck [14]. We also detected HPV 18 more frequently with Linear Array and HPV 31 and 33 more frequently with PapilloCheck, whereas HPV 16 was found equally often with both tests. Accordingly, kappa values for HPV 16 were higher than for HPV types, namely, 18, 31, and 33. However, except for HPV 18 and 52, kappa values represent good to very good concordance of typing results with PapilloCheck and Linear Array.

\section{Conclusion}

Our data indicate low prevalence of oropharyngeal HPV infection in patients with high-grade cervical dysplasia. The low detection rate was confirmed by using two different commercial assays with largely consistent results of HPV detection and typing, but with some variation for particular HPV types. Comparative testing of larger numbers is required to identify HPV types prone to escape detection with particular assays.

\section{Data Sharing Statement}

Detailed test results of all specimens are available by emailing d.grimm@uke.de.

\section{Funding Sources}

This work was supported by Greiner Bio-One $\mathrm{GmbH}$ (Frickenhausen, Germany). HPV PCR test were provided at no costs without restriction to the study protocol.

\section{Authors' Contribution}

DG, LW, and CJB designed the study. JB, KP, CJB, OB, and IT collected the samples. DG, JB, KP, OB, IT, and TM performed data analysis. DG, BS, and TM wrote the manuscript. LW, BS, CJB, OB, IT, SK, and VM reviewed the manuscript.

\section{Conflict of Interest}

Donata Grimm: Study support: none; Honoraria: Roche, ESOP Linn Woelber Study support: MSD, GSK, Medac oncology, Vaccibody; Honoraria/travel

Katharina Prieske: Study support: none; Honoraria: Astra Zeneca

Barbara Schmalfeldt: Study support: none; Honoraria: Roche, Astra Zeneca support: Roche, Medac, Jenapharm, MSD

Sasha Kürti: Study support and Honoraria: none

Chia-Jung Busch: Study support: none; Honoraria BMS

Ingo Teudt: Study support: Asklepios Klinik Hamburg Altona; Honoraria: none

Oliver Brummer: Study support: Asklepios Klinik Hamburg Altona; Honoraria: Roche, MSD

Volkmar Mueller: Study support and Honoraria: none

Thomas Meyer: Study support: none; Honoraria: Roche, Cepheid

Acknowledgement. The authors greatly acknowledge the technical support of Ina Raasch and Katja Warncke.

\section{References}

1. Zur Hausen H. Papillomaviruses in the causation of human cancers - a brief historical account. Virology. 2009;384:260-5.

2. International Agency for Research on Cancer (WHO). Monographs on the evaluation of carcinogenic risks to humans. Volume 100B: Biological Agents. Chapter Human papillomaviruses. 2012. p. 255-314 Available from: http://monographs.iarc.fr/ENG/Monographs/vol100B/mono100B.pdf.

3. Schiffman M, Castle PE, Jeronimo J et al. Human papillomavirus and cervical cancer. Lancet. 2007;370:890-907.

4. Kjaer SK, Frederiksen K, Munk C, et al. Long-term absolute risk of cervical intraepithelial neoplasia grade 3 or worse following human papillomavirus infection: role of persistence. $\mathrm{J}$ Natl Cancer Inst. 2010;102:1478-88.

5. Stanley M. Immune response to sexually transmitted HPV infection. In: Sexually transmitted infections and sexually transmitted diseases. Gross G, Tyring SK, editor. Springer Verlag: Berlin-Heidelberg; 2011, p. 441-56.

6. Kreimer AR, Bhatia RK, Messeguer AL, et al. Oral human papillomavirus in healthy individuals: a systematic review of the literature. Sex Transm Dis. 2010;37:386-91.

7. Meyer MF, Huebbers CU, Siefer OG, et al. Prevalence and risk factors for oral human papillomavirus infection in 129 women screend for cervical HPV infection. Oral Oncol 2014;50:27-31

8. Woelber L, Breuer J, Meyer T, et al. Oral Human Papillomavirus in Women With High-Grade Cervical Intraepithelial Neoplasia. J Low Genit Tract Dis. 2017;21:177-183

9. Gravitt PE, et al. Improved amplification of genital human papillomaviruses. J Clin Microbiol. 2000;38:357-61.

10. Altman DG. Practical statistics for medical research. 1st ed. Cheapman and Hall CRC Press Oxford, 1991;1-611.

11. Eklund C, Forslund O, Wallin KL, Dillner J. Global improvement in genotyping of human papillomavirus DNA: the 2011 HPV LabNet international proficiency study. J Clin Microbiol. 2014;52:449-459.

12. Martin Hernan F, Sanchez-Hernandez JG, Cano J, et al. Oral cancer, HPV infection and evidence of sexual transmission. Med Oral Patol Oral Cir Bucal. 2013;18:e439-444.

13. Termine N, Giovannelli L, Matranga D, et al. Oral human papillomavirus infection in women with cervical HPV infection: new data from an Italian cohort and a metanalysis of the literature. Oral Oncol. 2011;47:244-50.

14. Schopp B, Holz B, Zago M, et al. Evaluation oft he performance oft he novel PapilloCheck HPV genotyping test vy comparison with two other genotyping systems and the HC2 test. J Med Virol. 2010;82:605-15. 
15. Halfon $\mathrm{P}$, Benmoura D, Khiri $\mathrm{H}$, et al. Comparison of the clinical performance of carcinogenic HPV typing of the Linear Array and PapilloCheck HPV screening assay. J Clin Virol. 2010;47:38-42.

16. Crosbie EJ, Bailey A, Sargent A, et al. The PapilloCheck assay for detection of high-grade cervical intraepithelial neoplasia. J Clin Microbiol. 2015;533553-3559.
17. Heard I, Cushieri K, Geraets DT, et al. Clinical and analytical performance of the PapilloCheck HPV-Screening assay using the VALGENT framework. J Clin Virol. 2016;81:6-11.

18. Van Doorn LJ, Quint W, Kleter B, et al. Genotyping of human papillomavirus in liquid cytology cervical specimens by the PGMY line blot assay and the SPF(10) line probe assay. J Clin Microbiol. 2002;40:979-83. 